\title{
CAMPANHA DE ORIENTAÇÃO, PREVENÇÃO E DIAGNÓSTICO PRECOCE DE TUMORES MAMÁRIOS EM CADELAS E PREVALÊNCIA DESSES TUMORES DIAGNOSTICADOS DURANTE A CAMPANHA REALIZADA NO MUNICÍPIO DE BLUMENAU-SC
}

\author{
(Orientation campaign, prevention and early diagnosis of mammary tumors in female \\ dogs and prevalence of these tumors diagnosed during the campaign in the city of \\ Blumenau-SC)
}

\author{
Ana Lúcia Pascoli ${ }^{1}$, Silvio L. Negrão, Luciano E. Oliveira, Marília G.P.A. Ferreira, Nazilton P. \\ Reis Filho, Andrigo B. de Nardi
}

\begin{abstract}
${ }^{1}$ Correspondência: anapascoli@hotmail.com
RESUMO: Foi realizada uma campanha de prevenção contra o câncer de mama em Blumenau/SC, onde foram examinadas 111 cadelas para palpação digital das mamas. Foi entregue aos proprietários um folheto informativo sobre neoplasias mamárias, fatores de risco e proteção, formas de diagnóstico e tratamento e demonstrado como fazer palpação digital das mamas em seus animais a fim de detectar a presença de nódulos e conscientizá-los sobre o diagnóstico precoce. Em relação aos resultados obtidos, cães sem raça definida foram os mais acometidos $3(5 / 29,41 \%)$, sendo o Poodle o segundo (3/17,64\%). Em relação a faixa etária, 35,29\% (6) dos animais tinham até 5 anos, $41,18 \%$ (7) tinham de 6-10, e 23,53\% (4), de 11-16 anos. Em relação a incidência de mamas acometidas, 3 animais (17,64\%) apresentaram tumores em M1, 1 (5,88\%) em M2, 5 (29,41\%) em M3, $5(29,41 \%)$ em M4 e $3(17,64 \%)$ em M5. Foi observado maior incidência em animais não castrados $(58,82 \% / \mathrm{n}=10)$ e em $41,18 \%$ dos castrados (7), 3 deles (17,65\%) recebiam anticoncepcional e 14 (82,53\%) não recebiam, 6 deles apresentaram pseudociese e $11(64,71 \%)$ nunca apresentaram. Em relação ao escore corporal, 5 animais $(29,41 \%)$ foram considerados normais de acordo com a validação de Laflamme (1997), 7 (41,18\%) apresentavam excesso de peso e $5(29,41 \%)$ eram obesos. A detecção precoce das neoplasias mamárias em cadelas é um fator positivo em relação ao prognóstico. Sabe-se que alguns fatores influenciam a incidência de neoplasias mamárias, sendo assim, podemos prevenir esses fatores e consequentemente diminuir a incidência da neoplasia. Para isso, campanhas contra o câncer de mama devem ser realizadas para que proprietários conheçam as formas de prevenção e examinem periodicamente seus animais.
\end{abstract}

Palavras-chave: câncer; cão; mamas; palpação

ABSTRACT: A prevention campaign against mammary cancer in dogs was performed in Blumenau/SC, where 111 female dogs were examined to check for mammary cancer for digital palpation. It was delivered to owners a folder with mammary cancer information, risk factors and protective actions about diagnosis and treatment. It was also shown in their own dogs how to make digital palpation to detect the presence of nodules and make them aware of early diagnosis. Regarding the 2 results, mongrel dogs (SRD) were the most affected $(5 / 29,41 \%)$, and Poodle the second $(3 / 17,64 \%)$. In relation to age, $35.29 \%$ (6) animals were up to 5 years old, $41,18 \%$ (7) were 610 , and $23.53 \%$ (4), $11-16$ years old. Regarding the incidence of affected mammary, 3 animals (17.64\%) had tumors in M1, 1 (5.88\%) in M2, $5(29.41 \%)$ in M3, $5(29.41 \%)$ in M4 and $3(17.64 \%)$ in M5. About the reproductive status, it was observed a higher incidence of mammary nodules in noncastrated animals $(58.82 \% / \mathrm{n}=10)$ and $41.18 \%$ of castrated animals $(7), 3$ of them $(17.65 \%)$ were as contraceptive pregnancy prevention method and $14(82.53 \%)$ did not receive, 6 presenting pseudopregnancy and $11(64.71 \%)$ did not. In relation to the body score, 5 animals $(29.41 \%)$ were considered normal, according to the validation of Laflamme (1997), 7 (41.18\%) overweight and 5 $(29.41 \%)$ obese. Early detection of mammary tumors in dogs, it's a positive factor in respect to prognosis. It's known that some factors influence the incidence of mammary tumors, so we can prevent these factors and thus reduce the incidence of cancer. For this, prevention campaigns against mammary cancer should be held more frequently so that owners know how to prevent it and also periodically review their animals.

Key Words: cancer; dog; mammary; palpation 


\section{INTRODUÇÃO}

Devido ao fato das neoplasias estarem associadas a alto índice de mortalidade e morbidade em cães, cada vez mais tem sido observada a necessidade da realização do diagnóstico precoce, bem como a instituição do tratamento adequado, objetivando elevar ao máximo a expectativa e qualidade de vida de animais com câncer.

Os tumores mamários podem surgir em qualquer uma das cadeias mamárias sendo comum a presença de múltiplos nódulos de mesmo tipo ou de tipos histológicos diferentes (Queiroga e Lopes, 2002; Oliveira et al., 2003; Helmlén, 2005; Cassali et al., 2013). Comumente apresentam-se como nódulos circunscritos com tamanho, consistência e mobilidade variável, podendo estar associados à ulcerações da pele e reações inflamatórias locais (Queiroga e Lopes, 2002; Cassali et al., 2011).

O aspecto anatômico mais importante a ser considerado é a existência de uma quantidade variável de conexões de vasos linfáticos e sanguíneos entre a cadeia mamária direita e a esquerda, assim como entre glândulas mamárias ipsilaterais adjacentes. Essa característica anatômica pode justificar a alta incidência de múltiplas mamas afetadas por essa neoplasia na espécie canina (Morrison, 1998).

Fatores predisponentes como raça, idade, sexo, nutrição, atividade hormonal, entre outros podem estar associados à etiologia das neoplasias mamárias em cães. Neoplasias mamárias em cães desenvolvem-se com mais frequência nas glândulas mamárias inguinais (Birchard e Sherding, 2003). De acordo com Rutteman et al.(2001) aproximadamente $2 / 3$ dos tumores mamários caninos envolvem o quarto e o quinto par de glândulas, sendo observado nestas a presença de maior quantidade de parênquima mamário, estando sujeitos a maiores estímulos hormonais.

O exame clínico deve seguir uma rotina padronizada, devendo-se realizar - levantamento mais completo do histórico do animal. A inspeção e palpação das duas cadeias mamárias constituem etapas primordiais do exame físico. Devem ser observados dados como simetria glandular, localização, apresentação, tamanho e contorno do tumor, além de possíveis reações inflamatórias ou ulcerações, galactorréia, condição corpórea do paciente e o envolvimento de linfonodos regionais e/ou evidências de metástase a distância (Brasil, 1998; Misdorp et al., 1999; Zuccari et al., 2001; Cassali et al., 2013).

Exames como a histopatologia são indispensáveis para o diagnóstico definitivo, podendo ser possível detectar sua origem, grau de malignidade, além de permitir estabelecer 0 prognóstico (Misdorp, 2002; Cassali et al., 2013).

Para determinar o estadiamento clínico deve-se realizar radiografias de tórax em três posições (VD, LD, LE) com o objetivo de detecção de possível doença metastática pulmonar. Lesões pulmonares a partir de 6 a $8 \mathrm{~mm}$ podem ser visualizadas pela radiografia, enquanto que as menores que $6 \mathrm{~mm}$ apenas pela tomografia computadorizada. Exames ultrassonográficos de abdomen também devem ser realizados para investigação de metástases a distância (Cassali et al.,2011). O sistema TNM (Tumor, Linfonodo/do inglês node, Metástase) é o mais aceito para avaliação do estadiamento clínico, este foi estabelecido pela Organização Mundial da Saúde (OMS), e modificado para avaliação dos tumores mamários caninos. Esse sistema classifica o $\mathrm{T}$ como tumor primário, onde: T1- quando tumor é menor que $3 \mathrm{~cm}$, T2- tumor entre 3 a $5 \mathrm{~cm}$ e T3- tumor maior que 5 
cm. O N está relacionado aos linfonodos regionais, onde: No- alterações não evidentes, N1-alteração em linfonodo ipsilateral e N2- alterações em linfonodos bilaterais e a letra $M$ do sistema TNM está relacionada a metástases à distância, onde: M0metástase ausente e M1- presença de metástase.

De acordo com Misdorp (2002), Fossum (2013) e Cassali et al. (2013) a cirurgia é o tratamento de eleição para os tumores mamários benignos e malignos porém é contraindicada em casos de carcinomas inflamatórios. Animais com alto risco de desenvolver lesões metastáticas (Bergman, 2007; Gustafson e Page, 2013), com recidiva tumoral ou com metástase a distância possuem indicação de terapias adjuvantes baseadas no uso de quimioterapia antineoplásica (Gustafson e Page, 2013).

Em alguns estudos, os autores recomendam o acompanhamento e o reestadiamento da paciente após a mastectomia por até 2 anos. Exames físicos e radiográficos devem ser realizados periodicamente a fim de revelar recidivas ou metástases (Simon et al., 2006).

A neoplasia mamária é uma das causas mais comuns de metástases pulmonares em cadelas. A demora pela procura de assistência veterinária, certamente contribui com os avançados estadios da doença em que os animais são encontrados no momento do diagnóstico. Das neoplasias malignas mamárias nos cães, 25 a 50\% delas apresentam metástases antes da cirurgia (Cassali, 2000; Cassali et al., 2011).

Estudos apontam os benefícios da castração precoce, levando ao reconhecimento de que a ovariohisterectomia seja um dos melhores métodos de prevenção do desenvolvimento de neoplasias mamárias malignas (Rutteman e Kirpensteijn, 2003).

O presente estudo teve como objetivo realizar uma campanha de orientação, prevenção e diagnóstico precoce de tumores mamários em cadelas examinadas durante um evento de conscientização realizado na cidade de Blumenau/SC, e desta forma identificar a prevalência de tumores mamários de acordo com a raça e a faixa etária, quais as mamas foram as mais acometidas e relacionar a ocorrência de tumores mamários com a castração, utilização de terapia hormonal contraceptiva, histórico de pseudociese e obesidade.

\section{MATERIAL E MÉTODOS}

A campanha de prevenção contra - câncer de mama em cadelas foi realizada durante o evento denominado 17ํㅡㄹ Cãominhada que ocorreu na cidade de Blumenau, SC em abril de 2014. Os proprietários que passeavam no local foram convidados a participar da ação, sendo que os mesmos autorizaram a realização de exames físicos além da utilização dos dados obtidos durante a anamnese.

Foram examinadas 111 cadelas aleatoriamente, sem definição préestabelecida de raça e idade. Por meio de anamnese e exame físico foram coletados dados como raça, idade e histórico reprodutivo (castração, utilização de terapia hormonal e pseudogestação) e escore corporal, sendo os dados registrados em ficha clínica.

Quando nódulo mamários eram identificados, estes foram mensurados com auxílio e um paquímetro e e classificandos como T1 quando tumor era menor que $3 \mathrm{~cm}$, T2 de 3 a $5 \mathrm{~cm}$ e T3 quando era maior que $5 \mathrm{~cm}$ (Figura 1). 


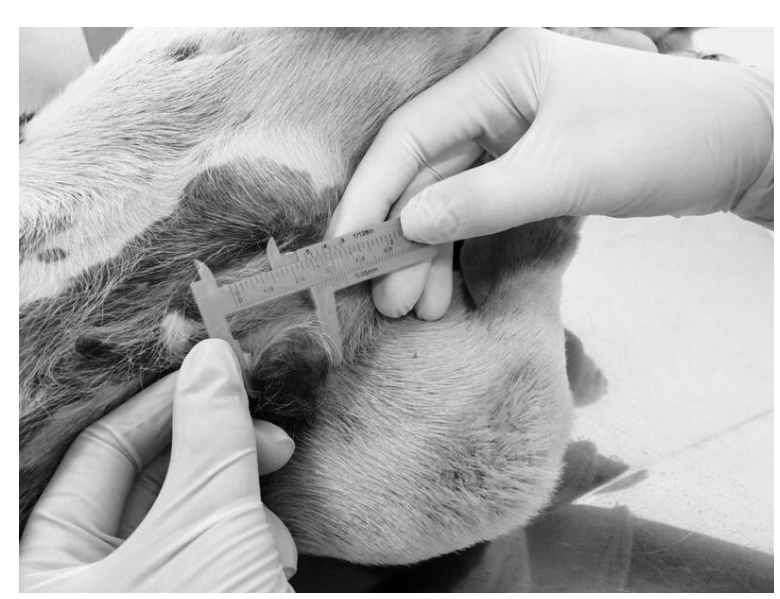

Figura 1 - Mensuração do tamanho do tumor com auxílio de paquímetro.

Os proprietários dos animais com tumores mamários foram aconselhados a encaminhar seus animais à um médico veterinário para a realização dos procedimentos necessários e adequado diagnóstico, estadiamento e tratamento. Além disso, foi entregue aos proprietários um folheto informativo sobre as neoplasias mamárias, fatores de risco e de proteção, além das formas de diagnóstico e tratamento. Também foi demonstrado aos proprietários como fazer palpação digital das mamas de seus cães, e recomendado a realização da palpação de forma periódica, a cada dois ou três meses, a fim de detectar a presença de tumores precocemente, favorecendo o prognóstico da doença.

Os resultados foram organizados em tabelas em uma planilha do Microsoft Office Excel 2007, contendo frequências e medidas descritivas com variáveis qualitativas. Os dados foram importados e tabulados pelo EPI INFO 7, onde foi possível determinar a prevalência das neoplasias bem como fazer associações com outras variáveis. Para realização das associações foi utilizado o teste de quiquadrado com nível de significância de 5\%.

\section{RESULTADOS E DISCUSSÃO}

Dos 111 animais examinados, observou-se a presença de tumores mamários, em 17 deles (15,31\%). Em relação à prevalência de tumores mamários de acordo com a raça, foi verificado que os cães sem raça definida (SRD) foram os mais acometidos $(n=5 / 29,41 \%)$, seguidos por cães da raça Poodle ( $n=3 / 17,64 \%)$, Shih tzu, Labrador ( $n=2 / 11,76 \%$ ) e Pinscher, Lhasa Apso, Dálmata, Dachshund e Chihuahua $(n=1 / 5,88 \%) \quad$ conforme demonstrado na figura 2 .

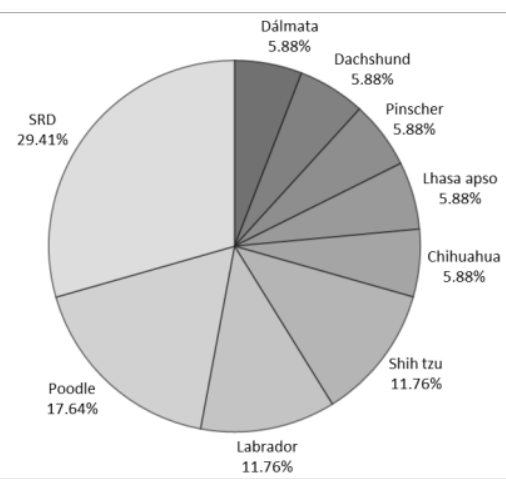

Figura 2 - Prevalência de tumores mamários de acordo com a raça.

A maior incidência de tumores foi observada em cães SRD 29,41\% e da raça Poodle $17,64 \%$, corroborando com Zuccari (1999) e Ferreira et al. (2003) que afirmam que no Brasil as neoplasias de mama ocorrem com mais frequência em animais SRD, devido ao fato de haver o predomínio de cães mestiços em nosso país. Fossum (2013) cita o Poodle, Boston Terrier, Fox Terrier e Daschund como raças com maior prevalência, corroborando ao observado no presente estudo no qual observou-se cães da raça Poodle como a segunda raça mais frequente.

Em relação a faixa etária, observou-se que $35,29 \%(n=6)$ dos pacientes com neoplasias mamárias apresentavam idade de até 5 anos. A faixa etária mais prevalente foi de 6 a 10 anos, totalizando $41,18 \%(n=7)$. Foram também verificadas que $23,53 \%(n=4)$ dos animais tinham idade variando entre 11 a 16 anos (Figura 3). 
Neste estudo foram examinados poucos animais com faixa etária entre 11 e 16 anos, acredita-se que isso pode ser justificado pelo fato de ser uma caminhada que exige resistência física dos animais, o que poderia justificar a menor frequência nesta faixa etária. A faixa etária de maior incidência encontrada no presente estudo corrobora Queiroga e Lopes (2002) que afirmam que as neoplasias mamárias acometem na maioria das vezes cadelas com idades entre os 8 e os 10 anos, no entanto, podem surgir tumores malignos em cadelas com menos de 5 anos.

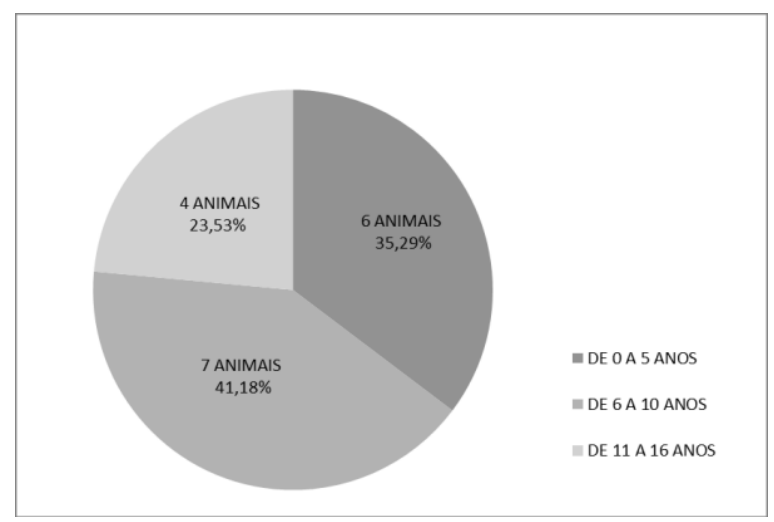

Figura 3 - Prevalência de tumores mamários de acordo com a faixa etária.

Com relação as mamas acometidas, um animal (5,88\%) apresentou acometimento em M1, 3 animais $(17,64 \%)$ apresentaram tumor em M2, 5 animais (29,41\%) em M3, 5 animais $(29,41 \%)$ em M4 e 3 animais $(17,64 \%)$ em M5 conforme demonstrado na figura 4 .

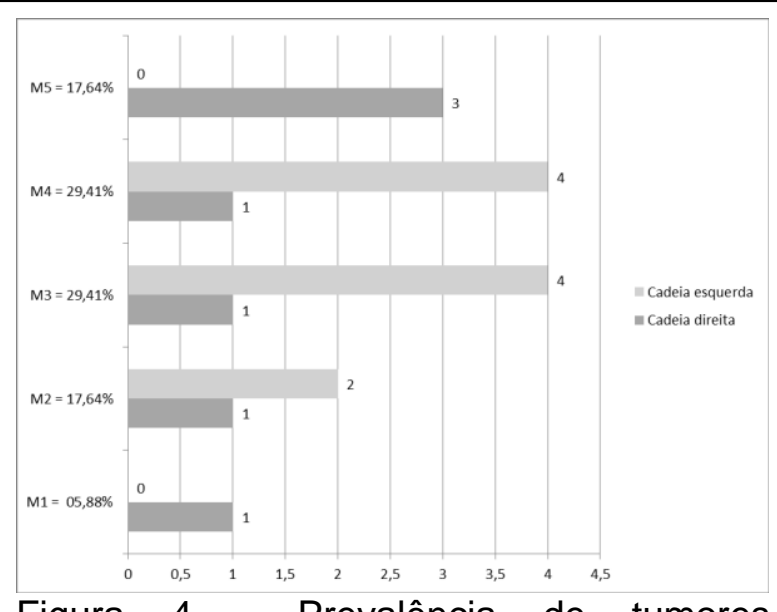

Figura 4 - Prevalência de tumores mamários de acordo com a mama acometida.

As mamas mais acometidas foram as M4 e M5 correspondendo a $47,05 \%$ do total de cães com tumores mamários, corroborando Birchard e Sherding (2003) que dizem que as neoplasias mamárias em cães desenvolvem-se com mais frequência em M5 (mamas inguinais) e com Cassali et al. (2011) que afirmam que as mamas mais acometidas são as M4 e M5 (inguinais e torácicas abdominais). Rutteman et al. (2001) afirmam que aproximadamente $2 / 3$ dos tumores mamários caninos envolvem as mamas M4 e M5, pois estes pares estão sujeitos a maiores estímulos hormonais devido a maior quantidade de parênquima mamário.

Em relação ao status reprodutivo dos animais, foi observado maior incidência de tumores mamários em animais não castrados $(58,82 \%)$ totalizando 10 animais e 41,18\% em animais castrados $(n=7)$ (Figura 5$)$. Dos animais sem tumores, $39(41,49 \%)$ eram castrados e $55(58,51 \%)$ não eram. 


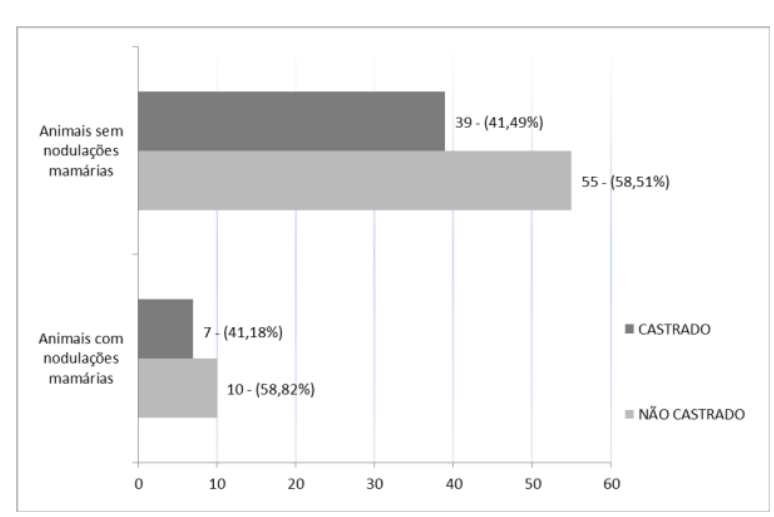

Figura 5 - Prevalência de animais castrados e não castrados com tumores mamários.

Não houve associação entre o status reprodutivo e a ocorrência de neoplasias mamárias, diferente do descrito por Slatter (2007) que afirma que a realização do procedimento de ovariohisterectomia (OHE) manifesta-se como um fator de proteção na formação dos tumores de mama principalmente se feito em idade precoce, pois a fonte hormonal (ovário) é removida pela cirurgia. Esse resultado pode ser justificado devido ao pequeno número de animais utilizados neste estudo e também por não ser possível a obtenção de dados em relação a idade de castração dos animais. Dos animais diagnosticados com tumores mamários, 3 deles $(17,65 \%)$ recebiam terapia anticoncepcional como método de prevenção de gestação e 14 deles $(82,53 \%)$ não recebiam (Figura 6). Não foi observado associação entre o uso de anticoncepcional e a presença de tumores mamários sendo que em relação aos animais sem tumores, 8 deles (8,51\%) recebiam e 86 (91,94\%) não recebiam $(P>0,05)$.

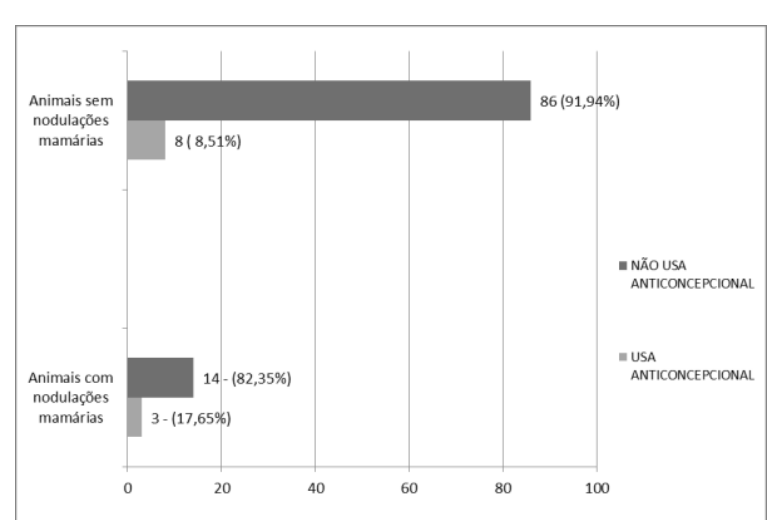

Figura 6 - Prevalência de tumores mamários relacionada ao uso de anticoncepcional.

Alguns pesquisadores encontraram associação entre 0 uso de anticoncepcional e neoplasia mamária, enquanto outros não encontraram efeito significativo na incidência destas, necessitando-se de mais estudos para se estabelecer com precisão o envolvimento do uso de anticontraceptivo na carcinogênese mamária (Mol et al., 1996).

Em relação aos animais com tumores mamários, seis deles apresentaram pseudogestação e 11 nunca apresentaram $(64,71 \%)$ conforme demonstrado na figura 7. O valor de $\mathrm{P}=0,0077$ mostra associação entre pseudociese e a presença de tumor. Dos animais sem tumores mamários, 84 deles $(89,73 \%)$ não apresentaram e 6 $(35,29 \%)$ apresentaram.

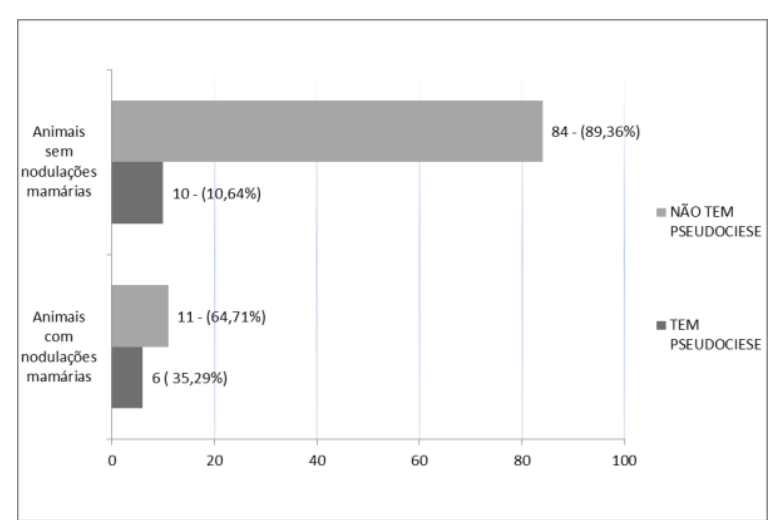

Figura 7 - Prevalência de tumores mamários relacionada a pseudociese.

Segundo Cirillo (2008) a
ocorrência da neoplasia está


relacionada principalmente a fatores hormonais, que apresentam um papel fundamental na carcinogênese mamária na espécie canina. O desenvolvimento e crescimento da glândula mamária encontram-se sob influência de hormônios ovarianos como o estrógeno e a progesterona, e a participação destes hormônios tem sido notada em numerosos contextos, tanto fisiológicos, como patológicos (Munson e Moresco, 2007).

Em relação ao escore corporal, baseado na validação de Laflamme (1997), 5 animais $(29,41 \%)$ foram considerados normais, 7 animais $(41,18 \%)$ com excesso de peso e outros 5 eram $(29,41 \%)$ obesos (Figura 8 ). Houve associação entre excesso de peso e obesidade e presença de tumores mamários $(P=0,0000014)$. Dos animais 15 sem tumores, 1 deles $(1,06 \%)$ foi considerado magro, 80 animais $(85,11 \%)$ normais, 10 animais $(10,64 \%)$ com excesso de peso e 3 animais $(3,19 \%)$ obesos.

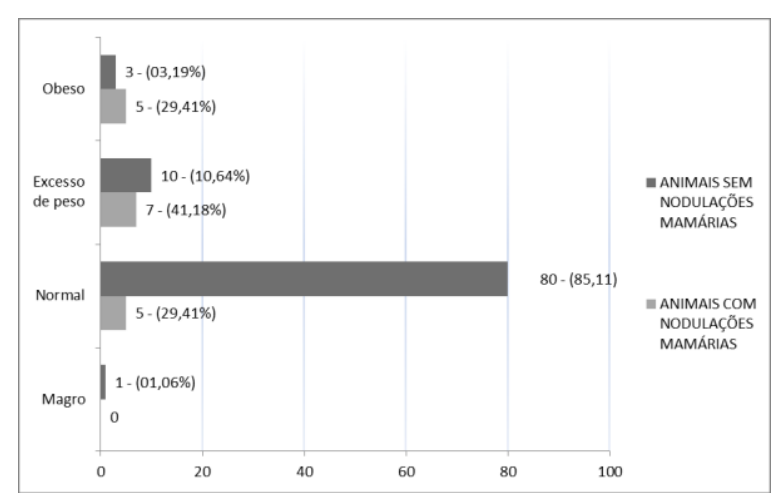

Figura 8 - Prevalência de tumores mamários relacionada ao escore corporal dos animais.

Neste estudo, o escore corporal foi a variável que mais teve associação com a incidência de nódulos dos animais que apresentavam tumores, $70,59 \%$ tinham excesso de peso e obesidade corroborando com Mathé (2000), que afirma que o risco relativo de câncer mamário está diretamente associado com o aumento do consumo de gordura. A obesidade afeta a expressão dos receptores de progesterona e pode agravar o "curso" do câncer de mama. Neste estudo foram avaliados dados referentes ao tipo de alimentação, se comercial ou caseira, porém não foram avaliados dados sobre a qualidade nutricional da alimentação. Alguns estudos demonstraram que 0 aumento da ingestão de gordura, durante o estágio de iniciação e promoção tumoral, pode aumentar o risco de desenvolvimento de neoplasias mamárias (Vorherr, 1987; Ray e Husain, 2001).

\section{CONCLUSÃO}

Concluiu-se neste estudo que a maior incidência de tumores mamários foi encontrada em cães sem raça definida (SRD), seguidos por cães da raça Poodle. A faixa etária mais acometida foi a de 6 a 10 anos e as mamas mais acometidas foram a $\mathrm{M} 3 \mathrm{e}$ M4. A maior parte dos animais com tumores tinham excesso de peso ou eram obesos, não eram castrados e a minoria utilizava anticoncepcional e já haviam tido algum episódio de pseudogestação.

Sabe-se que fatores como obesidade, castração, uso de hormônios, e pseudogestação possui relação com a incidência de neoplasias mamárias, sendo assim, podemos prevenir esses fatores e consequentemente diminuir a incidência desta neoplasia. Para isso, campanhas de prevenção contra o câncer de mama em cadelas devem ser realizadas com maior frequência para que os proprietários conheçam as formas de prevenção e também examinem periodicamente seus animais. Desta forma, é fundamental que os proprietários sejam orientados para que levem seus animais periodicamente ao médico veterinário, para que seja realizado o diagnóstico e o tratamento precoce das neoplasias mamárias, 
melhorando 0 prognóstico das pacientes, fazendo com estas possam atingir uma senilidade com mais saúde.

\section{NOTAS INFORMATIVAS}

Reservado ao parecer CEUA.

\section{REFERÊNCIAS}

BERGMAN, P.J. Mammary gland tumors. In: L. A. V. Conference (Ed.), The Latin American Veterinary Conference, Lima, Perú, 2007.

BIRCHARD, S.J.; SHERDING, R.G. Manual Saunders: clínica de pequenos animais. 2.ed. São Paulo: Roca, 2003.

BRASIL. Ministério da Saúde. Secretaria de Assistência à Saúde. Instituto Nacional de Câncer. União Internacional Contra o Câncer. TNM: classificação dos tumores malignos. 5.ed., Rio de Janeiro: Instituto Nacional de Câncer INCA, p.128-36, 1998.

CASSALI, G. D. Estudos morfológicos, imunohistoquímicos e citométricos de tumores mamários da cadela - aspectos comparativos com neoplasias da mama humana. 2000. Tese (Doutorado em Ciência Animal) Patologia, Escola de Veterinária, UFMG.

CASSALI, G.D. et al. Consensus for the Diagnosis, Prognosis and Treatment of

Canine Mammary Tumors. Brazilian Journal of Veterinary Pathology, v.4, n.2, p.153-180, 2011.

$\begin{array}{lll}\text { CIRILLO, J.V. Tratamento } & \end{array}$ mamárias em cadelas e gatas. Revista do Instituto de Ciências da Saúde, 2008.

FERREIRA, E.; PINTO, A.D.; AZEVEDO Jr, G.M.; CASSALI, G.D. Métodos de avaliação da proliferação celular em tumores mamários caninos: contagem de mitoses e imunohistoquímica para MIB-1. In: Encontro Nacional de Patologia

Veterinária, 2003, Botucatu. XI ENAPAVE, p. 270- 270, 2003.

FOSSUM, T.W. Cirurgia de Pequenos Animais, 4.ed., Rio de Janeiro: Mosby

Elsevier, 2013. p.809- 814.

GUSTAFSON, D.L.; PAGE, R.L. Cancer chemotherapy. In: WITHROW \&

MacEWEN's Small Animal Clinical Oncology, 5th edition, St Louis: Elsevier, 2013. Chapter 11.

HELLMÉN, E. Complex mammary tumors in the female dog: a review. Journal of Dairy Research, v.72, p.9097, 2005.

LAFLAMME, D.P. Development and validation of a body condition score system for dogs: a clinical tool. Canine Practice Journal, Santa Barbara, v.22, n.3, p.10-15, 1997.

MATHÉ, G. Obesity not only holds cardiovascular disease, it also hold tumors.

Biomedicine

\&

Pharmacotherapy Jounal, v.54, p.6768, 2000.

MISDORP, W.; ELSE, R.W.; HELLMÉN, E.; LIPSCOMB, T. Histological classification of mammary tumors of the dog and cat. Washington: Armed Forces

Institute of Pathology, 1999. p.18- 27.

MISDORP, $W$. Tumors of the mammary gland. In: MEUTEN, D.J. Tumors in domestic animals, 4th ed. lowa: Blackwell Publishing, 2002. p. 575-606.

MOL, J.A.; VAN GARDEREN, E.; RUTTEMAN, G.R.; RIJNBERK, A. New insights in the molecular mechanism of progestin-induced proliferation of mammary epithelium: induction of the local biosynthesis of growth hormone (GH) in the mammary gland of dogs, cats and humans. The Journal of 
Steroid Biochemistry and Molecular Biology, v.57, p.67-71, 1996.

MUNSON, L.; MORESCO, A. Comparative Pathology of Mammary Gland Cancers in Domestic and Wild Animals. Breast Disease Journal, v.28, p.7-21, 2007.

NELSON, R.W.; COUTO, C.G. Medicina Interna de Pequenos Animais. Rio de Janeiro: Guarabara Koogan, 2001.

OLIVEIRA, L.O. et al. Aspectos epidemiológicos da neoplasia mamária canina.,Orlando, Florida, USA: North American Veterinary Conference, Acta Scientiae Veterinariae, v.31, n.2, p.105-110, 2003.

QUEIROGA, F.; LOPES, C. Tumores mamários caninos, pesquisa de novos fatores de prognósticos. Revista Brasileira de Ciências Veterinárias, v.97, n.543, p.119-127, 2002.

RAY, G.N.; HUSAIN, S.A. Role of lipids, lipoproteins and vitamins in women with breast cancer. Clinical Biochemistry Journal, v.34, n.1, p.71-76. 2001.

RUTTEMAN, G.R.; WITHROW, S.J.; MacEWEN, E.G. Small Animal Clinical Oncology. 3th ed, Filadelfia: W.B. Saunders, 2001.

RUTTEMAN, G.R.; KIRPENSTEIJN, J. Tumours of the mammary glands. In: DOBSON, J.M.; LASCELLES, B.D.X. BSAVA Manual of Canine and Feline Oncology 2th ed. Gloucester: British Small Animal Veterinary Association, p. 234-242, 2003.

SIMON, D.; SCHOENROCK, D.; BAUMGÄRTNER, W.; NOLTE, I. Postoperative adjuvant treatment of invasive malignant mammary gland tumors in dogs with doxorubicin and docetaxel. Journal of Veterinary Internal Medicine, 20:1184-1190,
SLATTER, D. Manual de cirurgia de pequenos animais. 3.ed, São Paulo: Ed. Manole, 2007.

VORHERR, $\mathrm{H}$. Endocrinology of breast cancer. Maturitas Journal, v.9, n.2, p.113-122, 1987.

ZUCCARI, D.A.P.C. Contribuição ao estudo imunoistoquímico dos tumores mamários em cadelas. Jaboticabal, 121p. Dissertação (Mestrado em Patologia Animal) Faculdade de Ciências Agrárias e Veterinárias, Universidade Estadual Paulista, 1999.

ZUCCARI, D.A.P.C.; SANTANA, A.E.; ROCHA, N.S. Fisiopatologia da neoplasia mamária em cadelas revisão. Clínica Veterinária, v.32, p.5054, 2001.

2006. 